\title{
Percepción del estudiantado activo sobre las causas del abandono escolar en instituciones de secundaria de la Dirección Regional de Enseñanza de Cartago, Costa Rica
}

Ramírez Díaz, Jose Laurian; Castro Acevedo, Dinorah; Arrieta Quesada, Marcela; Redondo-Hernández, Mayra; Brenes Zamora, Manuel Alfonso

Percepción del estudiantado activo sobre las causas del abandono escolar en instituciones de secundaria de la Dirección Regional de Enseñanza de Cartago, Costa Rica

Revista Educación, vol. 42, núm. 2, 2018

Universidad de Costa Rica, Costa Rica

Disponible en: http://www.redalyc.org/articulo.oa?id=44055139005

DOI: https://doi.org/10.15517/revedu.v42i2.23574

Esta obra está bajo una Licencia Creative Commons Atribución-NoComercial-SinDerivar 3.0 Internacional. 


\section{Percepción del estudiantado activo sobre las causas del abandono escolar en} instituciones de secundaria de la Dirección Regional de Enseñanza de Cartago, Costa Rica

Regular Student's Perception by Causes of School Dropouts Colleges of Another Students, at Education Regional Management of Cartago, Costa Rica

Jose Laurian Ramirez Diaz [1]

Universidad Católica de Costa Rica, Costa Rica

DOI: https://doi.org/10.15517/revedu.v42i2.23574

joselaurian@gmail.com

Dinorah Castro Acevedo [2]

Ministerio de Educación Pública, Costa Rica

dinorahcastro70@hotmail.com

Recepción: 18 Marzo 2016

Aprobación: 20 Marzo 2018

Marcela Arrieta Quesada [3]

Ministerio de Educación Pública, Costa Rica

marcelarrieta@yahoo.com

Mayra Redondo-Hernández [4]

Ministerio de Educación Pública, Costa Rica

mayraredondo@hotmail.es

Manuel Alfonso Brenes Zamora [5]

Ministerio de Educación Pública, Costa Rica

malfonsobz65@gmail.com

\section{ReSUMEN:}

Son varios los estudios que se han hecho sobre abandono escolar, debido a sus repercusiones tanto para la persona como para la sociedad. En el ámbito costarricense se han creado programas de ayuda socioeconómica, pedagógica y psicosocial, que buscan mantener al estudiantado en las aulas, pero el fenómeno ha prevalecido en el tiempo. También se han implementado estrategias aisladas que pretenden disminuir los índices de exclusión escolar; pero su impacto no ha sido evaluado apropiadamente y tampoco se han socializado los resultados. Este documento presenta un estudio cuantitativo factorial comparativo que se realizó con 624 estudiantes de 20 colegios entre técnico profesionales y académicos de la Dirección Regional de Enseñanza de Cartago, tanto de modalidad diurna como nocturna, quienes expusieron su percepción sobre las causas del abandono escolar. Se busca proponer criterios técnicos de diagnóstico que sirvan como puntos de partida para implementar estrategias y acciones preventivas del abandono escolar, y que sean útiles como referencia para profesionistas en educación que se preocupan por esta problemática y desean ejecutar acciones de intervención y de prevención, en las cuales se consideren la motivación y el sentido de pertenencia del estudiantado al contexto educativo, como pilares de acciones preventivas y de gestión educativa.

\section{NotAS DE AUTOR}

[1] Magíster en Tecnología Educativa por la Universidad Estatal a Distancia. Orientador educativo. Profesor en Universidad Católica de Costa Rica y Profesional Asesor de Educación No Formal del Centro de Capacitación y Desarrollo (CECADES), Dirección General del Servicio Civil.

[2] Licenciada en Trabajo Social por la Universidad de Costa Rica. Trabajadora social de la Asesoría Regional de Orientación de Cartago, Ministerio de Educación Pública.

[3] Licenciada en Psicología por la Universidad Central. Psicóloga de la Asesoría Regional de Orientación de Cartago, Ministerio de Educación Pública.

[4] Máster en Psicopedagogía por la Universidad Florencio del Castillo. Orientadora Educativa. Trabaja en la Escuela Juan Vásquez de Coronado, Ministerio de Educación Pública.

[5] Máster en Administración Educativa por la Universidad Americana. Orientador de la Asesoría Regional de Orientación de Cartago, Ministerio de Educación Pública. 
Palabras Clave: Abandono escolar, repitencia, gestión educativa, motivación, orientación.

\section{Abstract:}

Many social studies have been made on scholar dropout because it's many implications for the people and the societies. The Costa Rican authorities has created socio-economic support programs as strategic to keep the students into education system but the phenomenon has prevailed over time. There have also been isolated implemented strategies that aim to decrease dropout rates but its impact has not been evaluated and the achievements are never socialized. This paper presents a study by comparative factorial quantitative technics was conducted with 624 students from 20 schools between technical professional secondary schools and academics colleges at Education Regional Management of Cartago province, both daytime and nighttime mode, and its exposed the perception of the causes of scholar dropout with the aim of proposing technical diagnostic items that serve as beginnings factor to implement strategies and preventive actions dropout in educational contexts and, which are useful as a reference for you education professionals who are concerned about this issue and want to perform actions to address and prevention, what consider the student's motivation and his sense of belonging to high school, as bases for prevention and educational management actions. KEYWORDS: School dropout, grade reprobate, educational management, motivation, counseling.

El abandono escolar es un fenómeno que tiene un alto costo social y económico, no solo por los recursos que se invierten en aras de la permanencia de estudiantes en las aulas, sino porque, con esto, las personas se privan de niveles educativos aptos de certificación para realizar trabajos con remuneración suficiente, que favorezcan el acceso a una mejor calidad de vida, entre otras situaciones más. Desde la perspectiva de la orientación, el fenómeno plantea distintas repercusiones en el desarrollo de las personas, que inciden en el proyecto de vida, la realización personal, la prevención de fenómenos como la violencia y otros más, por lo que las políticas preventorias del abandono escolar deben considerar la percepción estudiantil sobre las causas que repercuten en su prevalencia, así como las razones sociales que se encuentran involucradas en los ámbitos comunitarios e institucionales.

En este sentido, es importante mencionar que la Dirección Regional de Enseñanza de Cartago (DREC) está compuesta por instituciones de secundaria enriquecidas por la heterogeneidad: algunas pertenecientes a zonas rurales; otras marcadas por bajos índices de menor desarrollo social; instituciones académicas y colegios técnico profesionales de jornada diurna y nocturna, que atienden poblaciones adolescentes y adultas, entre otras más, de modo que las necesidades son influidas por la diversidad de la población que lo representan.

Según el Instituto Nacional de Estadísticas y Censos (INEC, 2012), Cartago es la provincia costarricense ubicada como la segunda con mayor tasa de asistencia a la educación regular $(69,1 \%)$, con una población que ronda el medio millón de habitantes, lo que implica un 11,4\% de la población a nivel nacional, con una edad promedio de 31 años. A los quince años la media del grado de escolaridad de la población cartaginesa ronda los 8,6 años, y por cada 100 mujeres coexisten 96 hombres (INEC, 2011). El 82,5\% de las personas con edad entre 12 a 17 años se dedica solo al estudio y, el 46,8\% de este grupo etario presenta una sobreedad de un año respecto al nivel de secundaria que debería cursar: dos años el 24,2\%, 14\% de tres años, y de cuatro años o más el restante 15,2\% (INEC, 2013).

La disposición emitida por la Dirección Regional a la Asesoría Regional de Orientación (ARO), plantea como directriz investigar las principales causas por las cuales se da el abandono escolar en secundaria, para proponer acciones a nivel regional e institucional que lo reduzcan, en al menos un punto porcentual. Con base en lo anterior, la DREC se planteó un estudio que tuvo como objetivo identificar las principales causas, percibidas por el estudiantado activo, que motivan el abandono escolar, con base en lo que conocen sobre situaciones de excompañeros y excompañeras, con el fin de involucrar a todos los agentes educativos en líneas de acción, de forma que armonicen con las expectativas del estudiantado y las demandas del sistema educativo. Ello busca mejorar la gestión y eficacia en las acciones preventorias, para disminuir los índices de dicho fenómeno y otros relacionados, como la tasa de repitencia, en distintas instancias y ámbitos educativos. 
Por lo anterior se planteó, como problema de investigación: ¿cuáles factores inciden en el abandono escolar, según la percepción del estudiantado activo de secundaria en la DREC? Este artículo muestra los resultados de dicho estudio, el cual fue realizado por personal de la Asesoría Regional de Orientación de Cartago, Costa Rica.

\section{ESTADO DE LA CUESTIÓN}

Los estudios sobre abandono escolar han sido varios, aunque no se han estructurado de forma longitudinal, sino que han propuesto análisis descriptivos, en su mayoría. A nivel global, el abandono escolar es un fenómeno de importante atención en el cual, además de múltiples causas relacionadas, parece existir una tendencia marcada por el desinterés estudiantil hacia los temas de estudio que propone la secundaria, así como una limitada proyección de los beneficios de la educación formal respecto al futuro personal y profesional de la población adolescente (Bolívar y López, 2009; Marina, 2011). Así mismo, según se interpreta de los resultados de la Organización para la Cooperación y el Desarrollo Económico (OCDE, 2016), en su Informe del Programa Internacional para la Evaluación de Estudiantes (PISA, 2016), los hombres parecen ser, de este grupo etario, quienes presentan mayor fracaso escolar, una de las razones que, con mayor incidencia, deriva en el abandono de las aulas (Calvo, 2011).

En el caso de América Latina, para inicios de este siglo se estimaba que el índice de abandono escolar rondaba el 37\% de adolescentes entre 15 a 17 años (Comisión Económica para América Latina y el Caribe, 2002). Entre las razones más frecuentes de dicho fenómeno se encuentran la maternidad y paternidad adolescentes, la necesidad de atender labores domésticas y, en concordancia con el panorama global, el desinterés por el estudio (Aunión, 2013).

En Costa Rica, el Programa Estado de la Nación (2015) es la principal referencia y fuente de consulta sobre las causas de la gestión educativa y los fenómenos que la involucran. También el Ministerio de Educación Pública ha desarrollado investigaciones al respecto (Jiménez y Gaete, 2010).

Grosso modo, los datos parecen mostrar que, desde inicios de este siglo, se ha consignado el abandono escolar como un fenómeno multicausal, donde se acentúan, como agentes activos, el nivel socioeconómico y las necesidades humanas de la población estudiantil (Rojas, 2000), aunque se destaca que los índices relacionados con este fenómeno han decaído en los últimos años en el país, pasando de 4.600 estudiantes en 2013 a 3.301 en 2014, con prevalencia mayor en los hombres con respecto a las mujeres (Cambronero, 2015; Fallas y Ross, 2014), lo cual es un hecho relevante, pero del que aún existen algunas conjeturas por resolver, especialmente aquellas referidas a las particularidades de distintos contextos sociales y comunidades específicas.

Datos del Quinto Informe del Estado de la Educación (Programa Estado de la Nación, 2015) indican que la motivación, el trato docente y la consideración de estudiantes en la labor institucional son causas altamente preventivas del abandono escolar, situaciones que deben considerarse para intervenir mediante acciones que disminuyan la prevalencia de dicho fenómeno. Esto refleja, a criterio particular, ciertas condiciones sobre el abandono escolar, las cuales se dan en distintos ámbitos, tal como se mencionó anteriormente: La proyección de los beneficios de la educación hacia el futuro en los ámbitos personal y profesional, así como el interés por los contenidos que integran el currículo académico deben ser considerados como ejes de análisis e intervención para su mejora, como estrategia de prevención.

\section{MARCO TEÓRICO}

El abandono escolar es un problema que preocupa mundialmente, pues limita el desarrollo humano, social y económico de las personas y los países (Marina, 2011; Ruiz, García y Pérez, 2014), y no es competencia 
exclusiva del personal docente o centro de estudios, sino que repercute a nivel social, precisamente por su prevalencia y complejidad (Castillo, 2006). Entre las causas que más afectan el abandono escolar están el bajo rendimiento, la desmotivación y las necesidades socioeconómicas como las principales (Programa Estado de la Nación, 2015). Esto coincide con las consideraciones de Jiménez y Gaete (2010) y Edel (2003), quienes explican que la desmotivación, relacionada a problemas socioeconómicos, el bajo rendimiento académico, el ausentismo y los problemas de conducta, entre otros, son las principales razones de dicho fenómeno, por lo que no es casualidad que el perfil de quien abandona las aulas describa a "un joven que no está motivado, falta mucho y no tiene la motivación familiar” (MEP y UNICEF, 2013, p. 84). También se propone al abandono escolar entre hermanos como otro riesgo potencial (MEP y UNICEF, 2013), así como la sobreedad (Programa Estado de la Nación, 2015).

La motivación es un componente necesario para prevenir el abandono escolar y se le considera adscrita al proyecto de vida (PV), el cual organiza la apertura del sujeto hacia su futuro, sus metas vitales y la toma de decisiones (D’Angelo, 2000), e involucra distintas dimensiones como la familia, la relación con docentes y el grupo, el bienestar percibido dentro de la institución educativa y la propia motivación (Pulido y Velasco, 2009) o, en caso de no hallarse en niveles adecuados, como limitante del desarrollo personal (Edel, 2003). Incluso el trabajo infantil o en personas menores de edad es otro de los elementos que comprometen la motivación para el estudio (Vargas y Slon, 2013). Es, además, un elemento de la calidad educativa y un indicador evaluador de la eficacia de la enseñanza (Jiménez, Terriquez y Robles, 2011), por lo que la prevención del abandono escolar apela, por un lado, a la mística docente, particularmente entendida como la habilidad del profesorado por despertar el interés en el estudiantado hacia el estudio, promoviendo, entre otras conductas, su permanencia en las aulas y, por otro, el apoyo familiar, como dos de sus mayores virtudes (Programa Estado de la Nación, 2013). Por tanto, el sistema educativo requiere humanizarse para favorecer la permanencia del estudiantado (León, 2011), entendido esto como la implementación de habilidades de comunicación que impliquen estrategia, progreso y eficacia en la institución educativa, pues si esta no existirían los grupos humanos ni las sociedades, así como la permanencia de estudiantes en las aulas, a pesar de las vicisitudes que limiten su regular asistencia (Calderón, 2012; Saló, 2005).

Para que existan sociedades debe satisfacerse una necesidad básica que considera el sentido de pertenencia a un grupo $\mathrm{u}$ organización, el cual propone un componente subjetivo conformado por percepciones, valoraciones y disposiciones de cada individuo (NU CEPAL, 2007). Las instituciones educativas implican un pequeño universo de convivencia, donde se comparten experiencias, existen diferentes temas de comunicación, entre otras situaciones más, por lo que es importante que se promuevan procesos en los que sus estudiantes desarrollen un sentido de pertenencia, lo cual se convierte en un factor preventorio del abandono escolar (Brea, 2014).

De acuerdo con el criterio de Tinto (2012), la retención de estudiantes en los sistemas educativos requiere de algunas condiciones, tales como:

- La expectativa del estudiantado sobre sí mismo, pues a mayor nivel, más probabilidad de que no se dé el abandono escolar.

- Soporte al estudiantado, el cual mantenga un interés latente en los logros e integre dimensiones como la académica, social y, en caso de ser necesario, económica para que este se mantenga en las aulas.

- Evaluación y realimentación, especialmente en los primeros años o antes y durante períodos importantes de transición, en los cuales se da un proceso de ajuste y adaptación al contexto educativo.

- Integración, la cual es considerada, por dicho autor, como la condición más importante para el mantenimiento del estudiantado en las aulas, y trata sobre las relaciones interpersonales y condiciones sociales que se gestiona en el centro educativo, tanto para el personal que en él labora, como para sus discentes. 
Lo anterior plantea, según Tinto (2012, citado por England, 2012), un concepto de persistencia del estudiantado, inscrito a la teoría de retención que él propone, que "las decisiones de los estudiantes de quedarse o dejar las instituciones se ve afectada por los niveles de conexión que ellos tienen con dicha institución educativa, tanto en el área académica como social" (p. 37). De este modo, si bien todo parece indicar que las causas que incentivan el abandono escolar son varias, también existen condiciones para que el estudiantado permanezca activo en las instituciones de secundaria y, todo apunta a que estas pueden ser identificas y considerarse en políticas y acciones de prevención.

A nivel nacional, la sobreedad escolar se concentra especialmente en el III ciclo, con un promedio de dos años (Programa Estado de la Nación, 2015), en tanto estas condiciones, aunadas a otras como la desmotivación por los estudios, la repitencia y el bajo rendimiento escolar, se constituyen en factores que favorecen dicho fenómeno. Por tanto, se consideró preciso identificar los elementos de mayor incidencia en el abandono escolar, de acuerdo con la percepción de estudiantado activo, y proponer incentivos y principios de líneas de intervención que disminuyan el número de estudiantes que cada año decide abandonar las aulas, resguardando una perspectiva disciplinaria desde la orientación, la cual está circunscrita como servicio dentro del sistema educativo formal y que Bisquerra (2001, citado por Ureña y Robles, 2014) puntualiza como un proceso de ayuda que tiene, como una de sus metas en el ámbito educativo, fortalecer los abecés relacionados al éxito escolar, para fomentar el desarrollo vital del sujeto.

Es importante considerar las características propias de las personas según su edad. En este sentido, Papalia, Duskin y Martorell (2012) mencionan que la adolescencia se presenta entre los 12 a 19 años, la adultez joven entre los 20 a 40 años y, la adultez media, se extiende desde los 40 hasta los 65 años.

\section{Procedimientos metodológicos}

El estudio tuvo un diseño cuantitativo de tipo explicativo, que analizó factorialmente la percepción de estudiantado activo sobre las causas que inciden en el abandono escolar y, consideró la relación entre las dimensiones planteadas, una característica esencial de este tipo de estudio (Hernández, Fernández y Baptista, 2014). La muestra fue de tipo no probabilística, compuesta por un total de 624 participantes: 347 (55,6\%) varones y $276(44,2 \%)$ mujeres. Los grupos de edad oscilaron entre adolescentes, personas adultas jóvenes y adultas medias. Esta se distribuyó entre un total de 20 colegios: 13 diurnos y 7 nocturnos; igual relación se dio entre instituciones académicas y técnico profesionales respectivamente. La muestra comprendió a todos los años académicos (séptimo a undécimo), así como el duodécimo año en los colegios técnico-profesionales. La Tabla 1 sintetiza las características de la muestra, con base en los criterios anteriores. 
TABLA 1

Grupos etarios según género

\begin{tabular}{lllll}
\hline Grupo etario & & Masculino & Femenino & Total \\
\hline Adolescentes & Frecuencia & 287 & 213 & 500 \\
\cline { 2 - 5 } & Porcentaje & $82,7 \%$ & $77,5 \%$ & $80,4 \%$ \\
\hline \multirow{2}{*}{ Adultos/as jóvenes } & Frecuencia & 56 & 60 & 116 \\
\cline { 2 - 5 } & Porcentaje & $16,1 \%$ & $21,8 \%$ & $18,6 \%$ \\
\hline \multirow{2}{*}{ Adultos/as medios/as } & Frecuencia & 4 & 2 & 6 \\
\cline { 2 - 5 } & Porcentaje & $1,2 \%$ & $0,7 \%$ & $1,0 \%$ \\
\hline \multirow{2}{*}{ Total } & Frecuencia & 347 & 275 & 622 \\
\cline { 2 - 5 } & Porcentaje & $100,0 \%$ & $100,0 \%$ & $100,0 \%$ \\
\hline
\end{tabular}

Nota: Elaboración propia del grupo autor (2017).

Sobre el procedimiento para recabar la información, profesionales de los Departamentos de Orientación de los colegios participantes fueron quienes administraron el cuestionario a estudiantes, el cual fue diseñado por personal de la Asesoría Regional de Orientación de la DREC. El instrumento se compuso de 17 preguntas sobre información general (género, edad, abandono escolar, entre otros), y una escala de Likert de 54 reactivos distribuidos en cuatro dimensiones: (1) apoyo emocional, (2) intervalos de estudio, (3) beneficio del aprendizaje y (4) gestión educativa. Para el análisis factorial y la ratificación de la validez y confiabilidad se utilizó el programa informático Statistical Package for the Social Sciences (SPSS). Dio como resultado una medida KMO de ,769 y una prueba de Bartlett que alcanzó $\mathrm{p}=, 000$, por tanto, las variables se correlacionan adecuadamente. En relación con las dimensiones planteadas para el análisis, el Alfa de Cronbach mostró un grado de fiabilidad que promedió ,757. La varianza explicada sumó $53,76 \%$, con lo que se sitúa dentro de un rango adecuado.

\section{Presentación y ANÁLisis de Resultados}

Como primer aspecto, cabe indicar que la repitencia se presentó en un total de 371 participantes (59,5\%), la cual es mayor para los hombres, ya que 211 (61,3\%) varones presentaron esta condición en comparación a 159 (57,8\%) mujeres. Así mismo, el año que presenta un mayor índice de repitencia para ambos géneros es séptimo con $131(37,2 \%)$ personas, seguido de octavo con 88 (13,11\%) participantes. Entre las causas del abandono escolar, el bajo rendimiento es la más recurrente, donde el $42,4 \%$ de los varones y $47,5 \%$ de las mujeres lo señalaron como causal.

En el estudio presente, los varones presentaron una tendencia mayor hacia el trabajo como medida para paliar las necesidades, pero muestran un menor índice de ayuda económica respecto a las mujeres en una relación del $40 \%$ y 52\%, respectivamente. Otra razón que motiva la exclusión estudiantil es el desinterés, situación que muestra mayor incidencia entre los varones por hasta 5 puntos porcentuales más respecto a las mujeres. Al respecto, la Tabla 2 muestra las razones por las cuales, según estudiantado activo consultado, sus excompañeros y excompañeras abandonaron el colegio. 
TABLA 2

Causas de abandono escolar según género

\begin{tabular}{|c|c|c|c|c|}
\hline & & Masculino & Femenino & Total \\
\hline \multirow[t]{2}{*}{ Rendimiento } & Frecuencia & 28 & 19 & 47 \\
\hline & Porcentaje & $31,1 \%$ & $26,0 \%$ & $28,8 \%$ \\
\hline \multirow[t]{2}{*}{ Económicos } & Frecuencia & 12 & 13 & 25 \\
\hline & Porcentaje & $13,3 \%$ & $17,8 \%$ & $15,3 \%$ \\
\hline \multirow[t]{2}{*}{ Personales } & Frecuencia & 13 & 11 & 24 \\
\hline & Porcentaje & $14,4 \%$ & $15,1 \%$ & $14,7 \%$ \\
\hline \multirow[t]{2}{*}{ Trabajo } & Frecuencia & 14 & 8 & 22 \\
\hline & Porcentaje & $15,6 \%$ & $11,0 \%$ & $13,5 \%$ \\
\hline \multirow[t]{2}{*}{ Desinterés } & Frecuencia & 15 & 6 & 21 \\
\hline & Porcentaje & $16,7 \%$ & $8,2 \%$ & $12,9 \%$ \\
\hline \multirow[t]{2}{*}{ Embarazo } & Frecuencia & 0 & 7 & 7 \\
\hline & Porcentaje & $0,0 \%$ & $9,6 \%$ & $4,3 \%$ \\
\hline \multirow[t]{2}{*}{ Ausentismo } & Frecuencia & 2 & 3 & 5 \\
\hline & Porcentaje & $2,2 \%$ & $4,1 \%$ & $3,1 \%$ \\
\hline \multirow[t]{2}{*}{ Traslado } & Frecuencia & 4 & 2 & 5 \\
\hline & Porcentaje & $4,4 \%$ & $2,7 \%$ & $4,2 \%$ \\
\hline \multirow[t]{2}{*}{ Drogas } & Frecuencia & 0 & 1 & 1 \\
\hline & Porcentaje & $0,0 \%$ & $1,4 \%$ & $0,6 \%$ \\
\hline \multirow[t]{2}{*}{ Total } & Frecuencia & 90 & 73 & 163 \\
\hline & Porcentaje & $100,0 \%$ & $100,0 \%$ & $100,0 \%$ \\
\hline
\end{tabular}

Nota: Elaboración propia del grupo autor (2017).

Respecto al grupo familiar, alrededor de 285 (48,3\%) participantes indicaron que viven con ambos progenitores y 229 (38,8\%) solo con su madre. Así mismo, el 82,11\% indicó tener hermanos o hermanas. En menor índice se presentaron familias compuestas, especialmente descritas por padrastros $(8,5 \%)$ y familias extendidas, con presencia de tíos o abuelos, pero en menor grado. Respecto al apoyo educativo, un total de 159 (40,9\%) participantes indicó que es la madre quien subsidia sus estudios, mientras que el apoyo por parte de ambos progenitores fue precisado por 116 (29,8\%) personas. Tíos, abuelos y otros parentescos también integran los recursos de apoyo familiar, pero en menor medida.

La sobreedad escolar promedio de la muestra se presentó en 1,66 años en III ciclo (séptimo, octavo y noveno), pero incrementa en el ciclo de Educación Diversificada (décimo y undécimo), donde algunos llegan a exceder hasta en 3,5 años su edad en relación con el año que cursan.

Para el estudio de las causas de abandono escolar, el análisis factorial se derivó en cuatro dimensiones. Primeramente, se consideró el apoyo educativo, entendido como el acompañamiento integral en el proceso de enseñanza aprendizaje, así como la atención a las dificultades individuales y la consideración de las emociones del estudiantado. El 49,6\% de participantes expusieron que la labor, en este sentido, es regular, mientras que el 35,4\% la percibieron como una dimensión que no es atendida, y solo un $15 \%$ que su atención es adecuada (véase Tabla 3). Es importante indicar que, en 5 de los 7 colegios nocturnos, el apoyo educativo es considerado como regular por la mitad de participantes. También en estas instituciones existe una menor cobertura de subsidios socioeconómicos (como becas) respecto a instituciones de modalidad diurna. 
TABLA 3

Apreciación del apoyo educativo según dicente

\begin{tabular}{lll}
\hline & Frecuencia & Porcentaje \\
\hline Deficiente & 210 & 35,4 \\
\hline Regular & 294 & 49,6 \\
\hline Adecuado & 89 & 15,0 \\
\hline Total & 593 & 100,0 \\
\hline
\end{tabular}

Nota: Elaboración propia del grupo autor (2017).

Sobre los intervalos de estudio, dimensión que consideró las horas dedicadas para el aprendizaje y la preparación para los exámenes y el rendimiento académico, el 57,9\% de participantes la valoraron como regular, un $24,3 \%$ consideró que su atención es adecuada y $17,8 \%$ como desatendida por completo (véase Tabla 4). El tiempo por semana que dedican al estudio son unas 1,36 horas en promedio. Sin embargo, las mujeres presentan una mayor inversión de tiempo semanal en este sentido (1,41 horas) respecto a los varones $(1,32)$. Solamente, un $6,7 \%$ de la muestra indicó que invierte tres o más horas para esta actividad en forma regular. Durante la semana de exámenes la media tiende a incrementar a 1,84 horas, aunque la diferencia por género se mantiene a favor de las damas, que invierten 1,94 horas a su estudio en comparación a las 1,75 de los hombres.

TABLA 4

Apreciación de Intervalos de tiempo para estudio semanal según estudiantes

\begin{tabular}{lll}
\hline & Frecuencia & Porcentaje \\
\hline Deficiente & 110 & 17,8 \\
\hline Regular & 358 & 57,9 \\
\hline Adecuado & 150 & 24,3 \\
\hline Total & 618 & 100,0 \\
\hline
\end{tabular}

Nota: Elaboración propia del grupo autor (2017).

La tercera dimensión supuso el beneficio del estudio analizado desde los hábitos de aprendizaje y el futuro profesional. El 85,2\% de la muestra consideró como un ámbito desatendido por completo y 7,4\% le calificó con una atención deficiente; igual porcentaje percibió que su atención es adecuada (véase Tabla 5).

TABLA 5

Valoración del beneficio del estudio según estudiantes

\begin{tabular}{lll}
\hline & Frecuencia & Porcentaje \\
\hline Deficiente & 528 & 85,2 \\
\hline Regular & 46 & 7,4 \\
\hline Adecuado & 46 & 7,4 \\
\hline Total & 620 & 100,0 \\
\hline
\end{tabular}


La gestión educativa consideró la forma en cómo el personal docente imparte lecciones, su forma de explicar los contenidos, aclaración de dudas y evaluación. En este sentido, 60,1\% de participantes consideró que es una dimensión de atención deficiente, $35,9 \%$ regular y $4 \%$ con una atención adecuada (véase Tabla 6).

TABLA 6

Valoración de la Gestión educativa según estudiantes

\begin{tabular}{lll}
\hline & Frecuencia & Porcentaje \\
\hline Deficiente & 374 & 60,1 \\
\hline Regular & 223 & 35,9 \\
\hline Adecuado & 25 & 4,0 \\
\hline Total & 622 & 100,0 \\
\hline
\end{tabular}

Nota: Elaboración propia del grupo autor (2017).

Es importante mencionar que 322 (52,8\%) estudiantes mencionaron que no conocen acciones concretas que se realicen en la institución para prevenir la exclusión, en contraste a 282 (46,2\%) que sí. Además 457 $(74,4 \%)$ integrantes de la muestra indicaron que en su institución existen grupos culturales, deportivos entre otros, aunque solo el 19,4\% pertenece a alguno, y el $48 \%$ no tiene interés en formar parte.

Finalmente, más de la mitad indicó que tiene alguna dificultad con alguna o varias materias de secundaria, y es matemáticas la que representa mayor conflicto según 259 (60,11\%) participantes, seguido por el inglés con el 38,15\% de la muestra (134 personas). En índices mucho menores, Estudios Sociales y las Ciencias se posicionaron como la tercera y cuarta materias con mayor dificultad para los varones, situación inversa en las mujeres, para quienes las Ciencias y los Estudios Sociales se posicionaron en el tercer y cuarto lugar de dificultad respectivamente.

\section{Discusión}

En lo que respecta al análisis de la información obtenida, el abandono escolar limita el desarrollo humano, social y económico de las personas. Es preocupante que, entre la percepción de estudiantado activo, parece que no existe un compromiso tangente por parte de las instituciones para mantenerles en las aulas, lo cual les ubica en una posición de desventaja hacia el futuro, pues el abandono escolar les implica un atraso en la conclusión de sus estudios, y las posibilidades para optar por un perfil que les permita el acceso a trabajos que propongan una remuneración que incida en la calidad de vida de forma positiva.

La motivación es un componente necesario que previene el abandono escolar. Como se explicó anteriormente, es parte de la calidad educativa y evalúa la eficacia de la enseñanza (Jiménez, Terriquez y Robles, 2011). La mística docente y el apoyo familiar son dos componentes preventorios de la exclusión escolar, de modo que las políticas institucionales deben enfocarse, además de enseñar al estudiantado, en ofrecerle un clima de convivencia adecuado, donde se sientan a gusto y, de este modo, se motiven por el aprendizaje, tal como plantea Tinto (2012) sobre la integración como elemento importante en la retención de estudiantes en las aulas. Las acciones, en este sentido, pueden ser varias, pero todas ellas requieren un elemento común: la integración activa del estudiantado en las actividades. Esto propone, tal como y se mencionó anteriormente, la humanización, entendida particularmente como la consideración activa del estudiantado como pilar de las gestiones y políticas educativas, para favorecer su permanencia en las aulas (León, 2011), al considerar a las personas que integran el proceso formativo, y no solamente los resultados cuantitativos de los procesos.

Es trascendental, por tanto, tratar alternativas que no solo valoren los conocimientos adquiridos, sino el nivel de satisfacción estudiantil con el desempeño de sus docentes y la organización en general. El 
Departamento de Orientación tiene un rol de alta relevancia como asesor en la promoción del desarrollo integral del estudiantado y del personal institucional, donde se considere la motivación como un elemento del planeamiento y ejecución de acciones dirigidas a la prevención del abandono escolar.

Entre los principales actores que previenen el abandono escolar, se encuentra el personal docente, ya que este no solo ejerce un rol tutor en los procesos, sino que propicia vínculos en los que el estudiantado se siente acompañado, no solo en el ámbito académico, sino también en el personal. Así mismo, en las organizaciones donde se requieran acciones que favorezcan la motivación, profesionistas en educación deben ejercer un rol de agentes de cambio y coordinar los recursos para que las acciones propuestas se materialicen, y evaluar el cumplimiento e impacto de los objetivos, tal y como proponen las demandas a la disciplina de orientación y organizaciones educativas en la actualidad (Boza, Toscano y Salas, 2007).

La comunicación entre docentes y estudiantes es primordial, no solo porque es necesaria en todas las relaciones humanas, sino porque incide en el sentido de pertenencia que cada persona desarrolla para con su grupo o institución, en este caso estudiantes, considerados aquí el grupo de pares, el clima organizacional, la relación con distintos agentes educativos, entre otros. El proceso formativo no debe limitarse solo a la asimilación del saber, sino a la visualización del sujeto como ser integral, una de las demandas más frecuentes por la parte dicente y un elemento preventorio del abandono escolar.

Se ha mencionado que la comunicación implica estrategia, progreso y eficacia en las organizaciones (Calderón, 2012; Saló, 2005). La comunicación debe proponer escenarios de entendimiento recíproco entre estudiantes y profesorado, que garanticen oportunidades de manifestarse y de ser objeto de escucha para ambas partes. Profesionistas en orientación y docentes tienen, como desafío, alentar espacios aptos para la comunicación, de modo que las necesidades de pertenencia sean manifestadas en forma clara y, se procure su satisfacción. En este sentido, la cohesión social es una búsqueda primaria de las personas y se manifiesta en la necesidad estudiantil de que se les tome en cuenta. El sentido de pertenencia a un grupo u organización es de suma relevancia, si se considera que los colegios, tal como se mencionó, implican un pequeño universo de convivencia, donde se comparten experiencias, existen diferentes temas de comunicación, entre otros (Brea, 2014). Al respecto, el profesorado y personal del colegio forman parte de ese universo: todo lo que comuniquen será material para procesar por parte de sus estudiantes; lo mismo sucede en caso inverso, de modo que también, es necesario que se revisen los mensajes que se dan a lo interno de los colegios, y la incidencia en la motivación del estudiantado para permanecer en la institución.

Otros factores relacionados con el abandono escolar son la repitencia y el fracaso escolar, los cuales inciden substancialmente en el PV. Se plantea, entonces, una tríada de factores: el bajo rendimiento académico, la repitencia y el abandono escolar, este último como consecuencia de la prevalencia de los dos primeros (Enríquez, Segura y Tovar, 2013).

Es importante que las políticas de prevención del abandono escolar consideren este triángulo para plantear acciones, en tanto no solo se demande al estudiantado la mejora del rendimiento académico, o se responsabilice exclusivamente al personal docente por la repitencia y situaciones similares, sino que se involucre a la totalidad de agentes educativos, desde la administración hasta la comunidad misma, en la propuesta de actividades que brinden un incentivo que trascienda la simple calificación; que promuevan la participación en labores que fomenten el sentimiento de pertenencia; que construyan legados para la institución y la comunidad; que consideren que la motivación se favorece mediante experiencias de calidad en el salón de clases y fuera de este (Navarrete, 2009), por tanto, puede verse como un transcurso de búsqueda, sostenimiento y regulación de acciones, que originan cambios en el entorno, en atención a las limitaciones individuales (Soriano, 2005).

Uno de los desafíos, para lo anterior radica en que solo 2 de cada 10 estudiantes integran grupos en sus colegios, pero solo la mitad de quienes no pertenecen a alguno tienen interés en adherirse, por tanto, la motivación no solo parece estar diezmada en este sentido, sino que las estrategias para lograrla parecen tener poco impacto. Sin duda, la motivación puede ser coadyuvada mediante grupos estudiantiles que propongan 
actividades que trasciendan la permanencia en el salón de clase, pero deben plantearse dinámicas congruentes a los intereses del grupo de discentes (mejor si son propuestas por el grupo mismo), de modo que el gusto por integrar algún colectivo aumente y, en consecuencia, la motivación.

En el estudio se encontró que existe una relación entre el trabajo infantil y el abandono escolar, situación que ha sido identificada en estudios previos. Parece existir una relación estrecha entre el abandono a las aulas y la responsabilidad con el hogar, ya que la segunda no permite contar con el tiempo suficiente para cumplir con los deberes académicos (Vargas y Slon, 2013). Además, preocupa el posible sesgo que existe entre el acceso a programas de ayuda entre hombres y mujeres ya que, para ambos, la relevancia de concluir sus estudios es la misma para acceder a mejores condiciones que eleven la calidad de vida, por tanto, debe revisarse, en futuros estudios, las causas de esta diferencia. Con respecto a esto, es importante recordar que, a nivel global, programas de evaluación como PISA, plantean que el fracaso escolar es mayor entre hombres, por lo que podría tratarse, aquí, de factores interrelacionados, que inciden en el abandono escolar para este grupo en particular. Parece que las condiciones materiales y económicas inciden directamente sobre la continuación de los estudios, pero las ayudas socioeconómicas no han sido suficientes para disminuir los índices de exclusión del sistema educativo, ya que la desmotivación y la falta de sentido de pertenencia tienen gran peso en estas decisiones, y se han mantenido hasta la fecha como causas relevantes, por lo que se recalca la necesidad de que docentes desarrollen habilidades para detectar los casos antes que se materialice el abandono escolar, con la asesoría y apoyo de profesionales en orientación, y el amparo de protocolos institucionales que integren a la totalidad de las personas, y no solo a unas cuantas.

En el país se han diseñado estrategias cuya meta es mantener a la juventud en las aulas mediante programas implementados en entornos de riesgo social, de este modo se les instituyen, a profesionistas en orientación, funciones de diagnóstico y seguimiento sobre causas que motivan el abandono escolar mediante protocolos de intervención, la asesoría, la mejora de los procesos y la evaluación de indicadores que guíen las políticas institucionales para retener al estudiantado (Jiménez y Gaete, 2010). Cabe destacar, en este sentido, el Programa Yo me apunto, gestado por el Ministerio de Educación Pública, que tiene como propósito el mantener al estudiantado en las aulas, previniendo, así, el abandono escolar, el cual constituye las políticas organizacionales para combatir dicha problemática (Ugarte, 2015) y que, a criterio particular, debe ser adaptado por docentes y personal orientador, según cada realidad institucional para garantizar un mayor éxito.

Empero, lo anterior puede motivar a que prevalezca un error común durante los últimos años, el cual, precisamente, trata sobre la división de funciones según el perfil profesional, lo que desvía la atención a propuestas que articulen objetivos comunes y evalúen el impacto, al proponer metas con base en ideales y no sobre las habilidades del personal docente y las organizaciones involucradas en los procesos de formación. Un ejemplo de esto son los programas de ayuda social y la evaluación de sus resultados, los cuales no se articulan en un trabajo en equipo que incorpore no solo la institución educativa, sino al grupo familiar y a la comunidad, por lo que solo proveen recursos sin que existan procesos en los que se instruya al estudiantado o a la organización en cómo administrarlos. Es necesario un cambio del paradigma de intervención, donde primero se consulte a los grupos dicentes sobre sus expectativas, intereses y habilidades y no plantear acciones con base en el criterio de personal de la administración, la docencia o la orientación, que puedan desviarse de las perspectivas del estudiantado y mermar su impacto.

Si bien se conocen las principales causas que alimentan la prevalencia del abandono escolar y el bajo rendimiento académico, el plantear acciones específicas para cada factor de riesgo mejoraría substancialmente la eficacia de las propuestas, al concentrar los esfuerzos en causas específicas, una a la vez. Así mismo, se reconoce que el abandono escolar entre hermanos o hermanas constituye un riesgo potencial de exclusión (MEP y UNICEF, 2013), de modo que se hace necesario, tal y como se mencionó, el diseño de estrategias que involucren a la familia desde un enfoque integral. No está en discusión el impacto de las políticas que cada colegio implemente, ya que cada uno debe velar por su evaluación, sino que se insiste en la necesidad 
de socializarlas para integrar a la comunidad estudiantil y las familias, incluso los colegios de la DREC entre sí, de modo que se propongan espacios de análisis y se vele por su mejora continua, para lo cual se adoptan acciones y estrategias que aumenten la efectividad de estas.

Otra problemática encontrada es que el abandono escolar y la repitencia retrasa la edad de conclusión de estudios, esto en los casos en los que el estudiantado decide regresar a las aulas, uno de los objetivos planteados por el programa Yo me apunto. La sobreedad de la muestra coincide con el índice nacional en cuanto a que la repitencia se concentra en el III Ciclo (Programa Estado de la Nación, 2015), pero la media en décimo y undécimo es mayor, ponderada en 3,5 para el caso de la DREC. Se apela aquí a considerar el impacto que este fenómeno puede tener en el desarrollo humano y las tareas para cada etapa del desarrollo, las metas individuales, las demandas de la sociedad y otros componentes más que pueden repercutir en una persona que, con 20 años, cursa el décimo año, por ejemplo. Deben considerarse, también, estrategias a futuro que intervengan este tipo de situaciones que, tal como se evidenció, son recurrentes en los colegios de la región.

De igual manera, se hace necesario mejorar el acompañamiento integral del estudiantado en su formación. Aunque el personal docente posee una función relevante en esto, debe involucrarse a todas las filiaciones de la organización. Es justo aclarar que el personal orientador ha aportado, de forma importante, al estudiantado en este sentido, por lo que la administración debe asumir un rol activo que preste oídos a toda la población involucrada en la formación, no solo al criterio de personal experto que, si bien es necesario, no es el único que existe. Además, consta una demanda por involucrar las emociones como parte de los procesos, lo cual se relaciona con la demanda estudiantil porque se les tome en en cuenta, mediante lo cual se estimula su sentido de pertenencia y, en consecuencia, es una maniobra eficaz de prevención del abandono escolar.

Es importante rescatar que, a nivel general, la mayoría de la muestra indicó que invierte poco tiempo para sus estudios, lo cual tiene obvias repercusiones en el rendimiento académico. Es poco probable que, con una inversión limitada en períodos de aprendizaje, se logre un desempeño y resultados óptimos, lo cual puede indicar un bajo nivel de motivación, por lo que es necesario que los grupos de estudiantes reciban información apropiada sobre la relevancia de los estudios, para lograr una adecuada calidad de vida, lo que se circunscribe a los ámbitos de intervención de la orientación; pero, además, docentes y demás agentes educativos deben involucrarse en forma activa y continua.

La atención sobre hábitos de estudio debe trascender el reparo exclusivo de la conducta, de modo que se amplíe a la consideración de estrategias de estimulación cognitiva y estructuración del tiempo para favorecer el PV y coadyuvar a la construcción del sentido de pertenencia estudiantil, de modo que también se brinde una evaluación cualitativa de autogestión sobre el proceso formativo. Se pueden implementar estrategias definidas sobre objetivos específicos, que planteen acciones para el logro paulatino, es decir uno a la vez, lo cual proporciona un escenario más favorable que incentive a la motivación. Respecto al PV, los procesos de orientación deben considerar la relación del estudio con el proceso de desarrollo y autorrealización. Aún queda una labor importante por hacer en este sentido, la que tradicionalmente es realizada por personal orientador; empero, parece que la demanda de estudiantes apuntala hacia el involucramiento de todos los actores educativos y la definición de estrategias sistémicas, que procuren resultados tangibles.

Por último, la dimensión que más diligencia demanda es la gestión educativa. Es necesario que se revisen las metodologías de enseñanza, la atención del personal docente a las dudas del estudiantado y las técnicas de evaluación. Se ha insistido en innovar hacia una estrategia que trascienda la evaluación sumativa, hacia otras que permitan al estudiantado crear legados en sus familias y la comunidad, de modo que evidencien el logro de los procesos en donde se involucra y le motive para continuar. En este sentido, es necesario considerar las expectativas por parte de discentes y mejorar los criterios de evaluación, con base en sus habilidades e intereses, lo cual no amerita una restructuración significativa de las matrices de los procesos, sino de adaptaciones que pueden plantearse en el salón de clase por la parte docente. Es necesario adaptar los métodos de prevención del abandono escolar a las necesidades del estudiantado, en vez de solo adecuarlas a los criterios externos o, dicho en otras palabras, tan importante es escuchar a los grupos de discentes como al criterio de los grupos 
expertos, para promover acciones eficaces que disminuyan los índices de abandono escolar a los niveles que se requieren.

\section{Conclusiones}

El abandono escolar es un fenómeno multicausal, lo cual ha quedado confirmado en los distintos estudios, incluido este, y es identificado así por el estudiantado. Del mismo modo, la motivación parece ser un elemento que le previene, pues la desmotivación parece tener más peso, al momento que una persona decide abandonar el colegio, por sobre otras causas, como la condición socioeconómica.

Así mismo, la motivación propone un parámetro para evaluar las acciones que se plantean para prevenir el abandono escolar y la calidad de la gestión educativa, ya que no solo apela a un factor aislado, sino que involucra procesos más complejos como la satisfacción de necesidades, el PV y el desarrollo de habilidades dentro del ámbito educativo, entre otros más. Pero debe considerarse la motivación como un incentivo no solo para el estudiantado, sino para el personal docente, que ejerce un rol sumamente importante en la permanencia de las personas en las aulas. Bien explica el Programa Estado de la Nación (2015), que una vez circunscrito al sistema educativo, ningún agente incide más en la persona que el perspnal docente.

Responsabilizar al profesorado por el bajo rendimiento académico no corresponde a una interpretación adecuada del fenómeno. Aunque es evidente que la relación de docentes con sus estudiantes tiene consecuencias en el continumm del segundo grupo, la familia tiene alta responsabilidad en el éxito escolar, imposible de satisfacer por otros actores. Las propuestas para prevenir el abandono escolar no deben limitarse a acciones aisladas o superpuestas a determinados perfiles ocupacionales, más bien debe analizarse cada causa del abandono y determinar su perfil específico, según la realidad de la institución, de la comunidad a la cual beneficia y las expectativas que cada estudiante tiene de estos elementos y de las metas que desea alcanzar, ya que parece que se ha equivocado la fórmula hasta ahora y es necesario un cambio en la intervención que se realiza en este sentido.

Entre las necesidades que más destacaron en el estudio, se rescata la necesidad que tiene el estudiantado en ser tomado en cuenta, esto al referir la opinión de personal experto mencionado sobre la retención de discentes en las aulas, la valoración de la gestión educativa y la proyecciónde los beneficios del estudio hacia futuro, según se interpreta particularmente. En este sentido, cada docente cuenta con todas las posibilidades para conocer a sus estudiantes, lo cual beneficia su labor, por tanto, esta demanda se dirige más hacia la administración y niveles jerárquicos fuera del salón de clase. Para prevenir la desmotivación y el bajo rendimiento académico debería innovarse hacia métodos que propongan trabajar en equipo y que busquen el logro sistémico de objetivos integrados en el bienestar común, donde el estudiantado pueda evaluar su legado por sí mismo, y se trascienda la evaluación meramente sumativa.

Poco vale la creación de grupos extracurriculares que no interesen a los estudiantes; generalmente porque las actividades propuestas no son congruentes con sus expectativas o intereses. Se requiere, entonces, un rol docente más avocado hacia la labor orientadora y, a profesionales en orientación con compromiso en las labores de investigación y diagnóstico, que propongan un conocimiento innovador y constante sobre las motivaciones del estudiantado, así como un ejercicio de continuo asesoramiento para crear y ejecutar acciones que estimulen la motivación, el éxito escolar y la permanencia en el sistema educativo. De igual manera, la lucha contra el trabajo infantil no debe cesar, ya que no solo demanda el sacrificio de un tiempo preciado para formar profesionales para el futuros, sino que involucra la calidad de vida de las personas que mantienen vínculos cercanos con estudiantes y el bienestar del país en general. En este sentido, puede aseverarse que la humanización de los procesos continúa siendo uno de los elementos más relevantes que motiva la permanencia de estudiantes en las aulas, lo que no es superado por factores como la ayuda económica, siquiera.

Si bien la percepción del estudiantado sobre el apoyo educativo debe mejorar, como parte de la formación integral debe ser una demanda de todo el personal docente el aumento substancial de los intervalos de tiempo 
que dedica el estudiantado a su aprendizaje. Esto no implica un equilibrio per se entre la motivación y el desempeño escolar, sino que podrían innovarse metodologías que propongan un uso del conocimiento de la vida cotidiana. Cuando el colegio consiga estimular que sus estudiantes operacionalicen lo aprendido en el salón de clase para resolver situaciones cotidianas, se habrá alcanzado uno de los objetivos que hasta la fecha no se ha podido lograr, lo cual no se da por falta de talento entre los agentes del sistema educativo, sino porque se han implementado las mismas estrategias de forma continua que parecen haber perdido su efectividad.

Es necesario, además, que dentro de los diagnósticos que realiza cada docente, también indague sobre la expectativa que tienen sus estudiantes con la metodología. La gestión educativa no pertenece solo a profesionales en educación, sino que implica varios factores que generalmente quedan por fuera al obviarse acciones sencillas como consultar sobre los intereses de quienes se benefician del proceso de enseñanza aprendizaje.

Es importante considerar, también, las repercusiones que tiene la sobreedad en las personas, no solo por el rezago, sino en la valoración de las tareas del desarrollo implicadas. $\mathrm{Al}$ respecto, es relevante prevenir el fenómeno desde el primer año de secundaria. Se insiste en el involucramiento de la familia del estudianado y el legado que desde su proceso educativo puede dar a su comunidad.

En resumen, las principales causas identificadas, con base en la información brindada por el estudiantado activo, en relación con las causas que originan el abandono escolar, parecen ser aquellas que limitan el sentido de pertenencia de los grupos discentes, o no promueven su motivación para el estudio, lo cual puede traducirse como obviar los aportes que el estudiantado puede brindar a los procesos y, por tanto, no tomarlo en cuenta dentro de la toma de deciones organizacional, situación que produce desmotivación y repercute en el rendimiento académico que ocasionalmente deriva en la repitencia. Parece, por tanto, que elevar el nivel de motivación e incentivar el sentido de pertenencia de estudiantes son dos elementos que previenen el abandono escolar y prevalecen, inclusive, por encima de problemáticas como, pueden ser, las limitaciones económicas.

De este modo, entre los factores que parecen incidir mayormente para que se dé el abandono escolar, se encuentran aquellos en los que el proceso formativo no logra adaptarse a las necesidades especifícas de cada institución, así como a las características de la población estudiantil que atiende, lo que ocasiona que, la consideración de políticas y estrategias de prevención se alejen de las expectativas de las generaciones jóvenes, pues dejan fuera sus intereses y no las involucra, con lo que disminuye su impacto real. Así, particularmente se interpreta que estudiantes con motivación y que se integren adecuadamente al contexto escolar se mantienen en las aulas, a pesar de las vicisitudes que puedan encontrar para ello.

\section{ReFERENCIAS}

Aunión, J. (7 de abril, 2013). La deserción escolar en América Latina ya no es solo cuestión de pobreza. Diario El País. Recuperado de https://elpais.com/sociedad/2013/04/07/actualidad/1365354378_499006.html

Bolívar, A. y López, L. (2009). Las grandes cifras del fracaso y los riesgos de exclusión educativa. Profesorado, 13(3), 51-78. Recuperado de http://www.ugr.es/ recfpro/rev133ART2.pdf

Boza, Á., Toscano, M. y Salas, M. (2007). ¿Qué es lo que hace un orientador?: Roles y funciones del orientador en educación secundaria. Revista de Educación, 21(9), 111-131. Recuperado de http://rabida.uhu.es/dspace/ bitstream/handle/10272/11243/Que_es_lo_que_hace_un_orientador.pdf?sequence=2

Brea, L. M. (2014). Factores determinantes del sentido de pertenencia de los estudiantes de Arquitectura de la Pontificia Universidad Católica Madre y Maestra, Campus Santo Tomás de Aquino (Tesis doctoral). Universidad de Murcia, Murcia, España. Recuperado de http://www.tdx.cat/bitstream/ handle/10803/284952/TLMBA.pdf?sequence

Calderón, Á. (10 de abril, 2012). Necesidad de la comunicación. El Universo. Recuperado de https:// www.eluniverso.com/2012/04/10/1/1366/necesidad-comunicacion.html 
Calvo, M. (23 de febrero, 2011). Fracaso escolar: Un problema principalmente masculino. El Confidencial. Recuperado de https://blogs.elconfidencial.com/espana/tribuna/2011-02-23/fracaso-escolar-un-problemaprincipalmente-masculino_727079/

Cambronero, N. (25 de marzo, 2015). Deserción escolar en colegios públicos cayó al 8,7\% el año pasado. Periódico La Nación. San José, Costa Rica. Recuperado de https://www.nacion.com/el-pais/educacion/desercion-encolegios-publicos-cayo-al-87-en-2014/BPQBP6HVG5HBZFL6X434REVNCY/story/

Castillo, M. (2006). Cómo evitar el fracaso escolar en secundaria. Madrid: Narcea.

Comisión Económica para América Latina y el Caribe. (Noviembre, 2002). Panorama social de América Latina 2001-2002. Recuperado de http://www.cepal.org/es/publicaciones/1213-panorama-social-americalatina-2001-2002

D’Angelo, O. (2000). Proyecto de vida como categoría básica de interpretación de la identidad individual y social. Revista Cubana de Psicología, 17(3), 1-13. Recuperado de http://pepsic.bvsalud.org/pdf/rcp/v17n3/08.pdf

Edel, R. (2003). Factores asociados al rendimiento académico. Revista Iberoamericana de Educación, 1(21), 1-21.

England, C. (2012). Teori\#a social cognitiva y teori\#a de retencio\#n de Vincent Tinto: Marco teo\#rico para el estudio y medicio\#n de la auto-eficacia acade\#mica en estudiantes universitarios. Revista Griot, 5(1), 28-49. Recuperado de http://revistas.upr.edu/index.php/griot/article/view/1774

Enríquez, C., Segura, A. y Tovar, J. (2013). Factores de riesgo asociados a bajo rendimiento académico en escolares de Bogotá. Investigaciones Andina, 26(15), 654-666. Recuperado de http://www.redalyc.org/articulo.oa? id $=239026287004$

Fallas, H.y Ross, A. (26 de mayo, 2014). Mística de docentes salvó a 6.000 colegiales de desertar. La Nación. Recuperado de https://www.nacion.com/gnfactory/especiales/2014/desercionestudiantil/index.html

Hernández, R., Fernández, C. y Baptista, P. (2014). Metodología de la investigación (6ª ed.). México D.F.: McGrawHill.

Instituto Nacional de Estadísticas y Censos. (2011). X Censo Nacional de Población y VI de Vivienda 2011. Resultados generales. San José: Autor.

Instituto Nacional de Estadísticas y Censos. (2012). X Censo Nacional de Población y VI de Vivienda. Indicadores provinciales. San José: Autor.

Instituto Nacional de Estadísticas y Censos. (2013). Costa Rica: Indicadores de educación y de contexto. San José: Autor.

Jiménez, A., Terriquez, B. y Robles, F. (2011). Evaluación de la satisfacción académica de los estudiantes de la Universidad Autónoma de Nayarit. Revista Fuente, 3(6), 46-56. Recuperado de http://fuente.uan.edu.mx/ publicaciones/02-06/8.pdf

Jiménez, W. y Gaete, M. (2010). Informe de investigación. Abandono (deserción) escolar en la enseñanza secundaria en Costa Rica, 2009-2010. San José, Costa Rica: MEP, Departamento de Estudios e Investigación Educativa. Recuperado de http://www.mep.go.cr/sites/default/files/DesercionSecundaria.pdf

León, F. J. (2011). Bienestar estudiantil. Significados que otorgan estudiantes y profesores. (Tesis de maestría). Universidad de Chile, Santiago, Chile. Recuperado de http://repositorio.uchile.cl/tesis/uchile/2011/cs-leon_f/ pdfAmont/cs-leon_f.pdf

Marina, J. A. (2011). El abandono escolar. Avances en Supervisión Educativa, 14, 1-5. Recuperado de https:// avances.adide.org/index.php/ase/article/view/467/311

Ministerio de Educación Pública y Fondo de las Naciones Unidas para la Infancia. (2013). Abandono escolar en la secundaria costarricense: Estudio de línea de base para los 100 centros educativos del ProEDUCA, 2012. San José, Costa Rica: Unicef. Recuperado de http://www.siteal.iipe.unesco.org/sites/default/files/crc-proeduca.pdf

Navarrete, B. (2009). La motivación en el aula. Funciones del profesor para mejorar la motivación en el aprendizaje. Innovación y experiencias educativas, 15, 1-9. Recuperado de https://archivos.csif.es/archivos/andalucia/ ensenanza/revistas/csicsif/revista/pdf/Numero_15/BELEN_NAVARRETE_1.pdf 
NU CEPAL. (2007). Cohesión social. Inclusión y sentido de pertenencia en América Latina y el Caribe. Santiago de Chile: CEPAL.

Organización para la Cooperación y el Desarrollo Económico. (2016). PISA 2015. Resultados clave (Documento digital). Recuperado de https://www.oecd.org/pisa/pisa-2015-results-in-focus-ESP.pdf

Papalia, D., Duskin, R. y Martorell, G. (2012). Desarrollo humano (12a ed.). México D.F.: McGraw-Hill.

Programa Estado de la Nación. (2013). Cuarto Informe Estado de la Educación. San José, Costa Rica: Autor. Recuperado de http://www.estadonacion.or.cr/informe-iv-estado-educacion

Programa Estado de la Nación. (2015). Quinto Informe Estado de la Educación. San José, Costa Rica: Autor.

Pulido, D. y Velasco, L. (2009). Proyecto de vida: Una alternativa para la prevención de la deserción escolar. Deserción escolar y proyecto de vida, 1-30. Recuperado de http://intellectum.unisabana.edu.co/bitstream/ handle/10818/2603/121977.pdf;sequence=1

Rojas, M. (2000). La deserción escolar en Costa Rica: un estudio de causas y consecuencias en una institución educativa. Diálogos. Revista Electrónica de Historia, 1(4), 1-26. Recuperado de https://revistas.ucr.ac.cr/index.php/ dialogos/article/view/6352/6055

Ruiz, R., García, J. y Pérez, A. (2014). Causas y consecuencias de la deserción escolar en el bachillerato: Caso Universidad Autónoma de Sinaloa. Ra Ximhai, 10(5), 51-74. Recuperado de http://www.redalyc.org/ pdf/461/46132134004.pdf

Saló, N. (2005). Aprender a comunicarse en las organizaciones. Madrid: Paidós .

Soriano, M. M. (2005). La motivación, pilar básico de todo tipo de esfuerzo. Revista de Relaciones Laborales, 9(1), 163-184. Recuperado de https://dialnet.unirioja.es/servlet/autor?codigo=261605

Tinto, V. (2012). Completing College: Rethinking Institutional Action. Chicago: University of Chicago Press. doi: https://doi.org/10.7208/chicago/9780226804545.001.0001

Ugarte, J. (28 de mayo, 2015). Con "Yo me apunto" el MEP pretende involucrar a todos los sectores sociales para disminuir la exclusión estudiantil. CRHoy.com. Recuperado de http://www.crhoy.com/archivo/con-yo-me-apunto-el-mep-pretende-involucrar-a-todos-lossectores-sociales-para-disminuir-la-exclusion-estudiantil/nacionales/

Ureña, V. y Robles, J. (2014). La orientación en el Ministerio de Educación Pública Costarricense. Actualidades Investigativas en Educacio\#n, 15(1), 1-19. Recuperado de http://www.scielo.sa.cr/pdf/aie/v15n1/ a34v15n1.pdf

Vargas, J. y Slon, P. (2013). Informe final ¿Protegen los programas sociales de la deserción educativa en Costa Rica? (Cuarto informe Estado de la Educación). San José: Costa Rica: Estado de la Nación. Recuperado de https:// www.estadonacion.or.cr/files/biblioteca_virtual/educacion/004/slon-y-vargas-desercion-educativa.pdf

\section{BY-NC-ND}

